\title{
Revisiting UML Class Relationship Recovery for Online Education
}

\author{
Dionysis Athanasopoulos \\ School of Electronics, Electrical Engineering, and Computer Science \\ Queen's University of Belfast, Northern Ireland, UK \\ D.Athanasopoulos@qub.ac.uk
}

\begin{abstract}
$U M L$ recovery has been a long-standing challenge for the software-engineering community. The complete recovery of UML class relationships needs the employment of both static and dynamic code analyses. However, the dynamic-code analysis is not usually applicable at the design time of programs and especially for incomplete programs in online education. To overcome this restriction, we propose a formally defined set of mappings between $U M L$ relationships and object-oriented relationships that are based on static-code analysis exclusively. We evaluate the precision and the recall of our mappings on student projects against ground-truth UML diagrams and against diagrams recovered by existing UML class recovery tools.
\end{abstract}

\section{Introduction}

The motivation of our research come from a real story. It all started a few weeks ago in the labs of a computerscience school. Amelia ${ }^{1}$, an undergraduate student, wanted in the context of a software-design module to take online feedback on her UML class diagrams ${ }^{2}$ that visualize the design of her Object-Oriented (OO) programs. Class diagrams describe the static structure of OO programs by showing the program's classes, fields, methods, and class relationships.

Amelia generally feels confident to build up a UML diagram only if she can map it to the source-code elements that implement the diagram. In other words, she prefers first writing (a skeleton of) her OO programs and then mapping them to UML diagrams via using her favorite integrated development environment, IDE (e.g., Eclipse ${ }^{3}$ ). The program that she has started developing today contains classes that are related to each other in various ways. Amelia found it difficult to build the diagram on her own and especially, to

\footnotetext{
DOI reference number: 10.18293/SEKE2021-170

${ }^{1}$ Please note that the persona names in our story are fake.

2 https://www.uml.org

$3_{\text {https: / / www.eclipse.org }}$
}

differentiate the usage of the various kinds of arrows that UML provides. In particular, she was confused while she was mapping the implementation-level relationships of her program to UML class arrows.

Thus, Amelia needed an online tool that takes as input her OO programs and outputs a visual medium for her programs. Such a tool should be quite precise with respect to the usage of the UML arrows. Moreover, the tool should be able to work on incomplete programs that cannot necessarily be executed. In other words, the tool should be based on the static-code analysis of OO programs. Amelia thought such a tool is a necessary classroom assistant in the era of online education that has recently stressed.

Luckily for Amelia, her module owner, Bob, suggested to her to use a freeware (e.g., ObjectAid ${ }^{4}$ ) that can be integrated with her IDE and recover UML diagrams from incomplete Java programs. Amelia was happy to see that the tool can draw UML diagrams by just dragging and dropping Java classes, providing a visual medium for Java programs. However, when she used the tool for her programs, she was concerned about the arrows used by the tool used for some Java class relationships in the recovered diagram. To double check the diagram, Amelia discussed her concerns with the module owner. Bob drew his own diagram and verified Amelia's concerns about the precision of the tool, as analysed in a next section of the current paper.

Overall, existing UML recovery tools that use staticcode analysis are not precise enough for online learning purposes. Moreover, the state-of-the-art research approaches that could be adopted for overcoming this limitation are not completely based on static-code analysis (e.g., [1, 2]) or they do not satisfy the lifetime and the share-ability object properties required for recovering the UML composition relationship (e.g., [3]).

We contribute an initial version of an automatic approach that takes as input an OO program and outputs the expected UML class relationships. To this end, we formally define the concepts of OO classifier, OO relationship, and UML relationship via using static-code syntactic analysis exclu-

\footnotetext{
${ }^{4}$ https://www.objectaid.com/home
} 
sively. We further propose a formally defined set of mappings between $\mathrm{OO}$ and UML relationships that satisfy the required lifetime and the share-ability properties ${ }^{5}$. We finally evaluate the precision and the recall of our mappings on existing student projects against ground-truth UML diagrams and against diagrams recovered by existing professional UML class recovery tools.

The rest of the paper is structured as follows. Section 2 presents the related research approaches. Section 3 defines the concept of OO relationship. Section 4 maps OO relationships to UML relationships. Section 5 evaluates the effectiveness of our approach. Finally, Section 6 summarizes our contribution and proposes future research directions.

\section{Related Work}

UML class diagrams represent OO classifiers (e.g., class, interface), fields, methods, and classifier relationships. The UML standard ${ }^{6}$ defines the following kinds of relationships between classifiers: dependency, inheritance, realization, association, aggregation, and composition. The association can be a directed or a unidirectional relationship.

We organize the existing approaches of the round-trip engineering between UML diagrams and $\mathrm{OO}$ programs into three categories. The first-category approaches generate source code from UML diagrams based on UML to OO mappings [4]. Other approaches recover business processes from UML sequence diagrams by using a set of heuristics [5]. The second-category approaches define consistency links between UML diagrams and source code $[6,7,8,9]$.

The third category includes reverse-engineering approaches that recover (parts of) UML diagrams from $\mathrm{OO}$ source code. [10] recovers UML use-case diagrams by using trace-ability links between use-case elements and classifiers. [11] recovers UML behaviour diagrams from source code by identifying patterns in the source code. [1, 2, 12] recover UML relationships by identifying mappings between UML and OO relationships. [13] apply heuristics to static and semantic analysis of Java classes.

Our approach belongs to the third category and is related to $[1,2,3]$. $[1,2]$ recover UML relationships via checking the following set of properties for objects: multiplicity, exclusivity, and lifetime. However, static and dynamic code analyses are used to confirm the properties.

$[14,3]$ recover composition relationships via checking the non-accessibility property for objects. To this end, $[14,3]$ check whether a reference to an object is exported by its owner object to a third-party object. However, [15] states that the definition of composition based on the nonaccessibility property is not consistent with the UML spec-

\footnotetext{
${ }^{5}$ We have left as future work the possible consideration of semantic code analysis (e.g., lexical analysis).

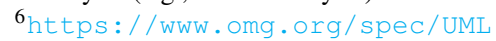

ification. [15] further states that the lifetime and the shareability properties are the properties that should be used for recognizing composition relationships. [15] specifies an OCL formalization of the above properties. However, the complete verification of the above properties needs both static and dynamic code analyses.

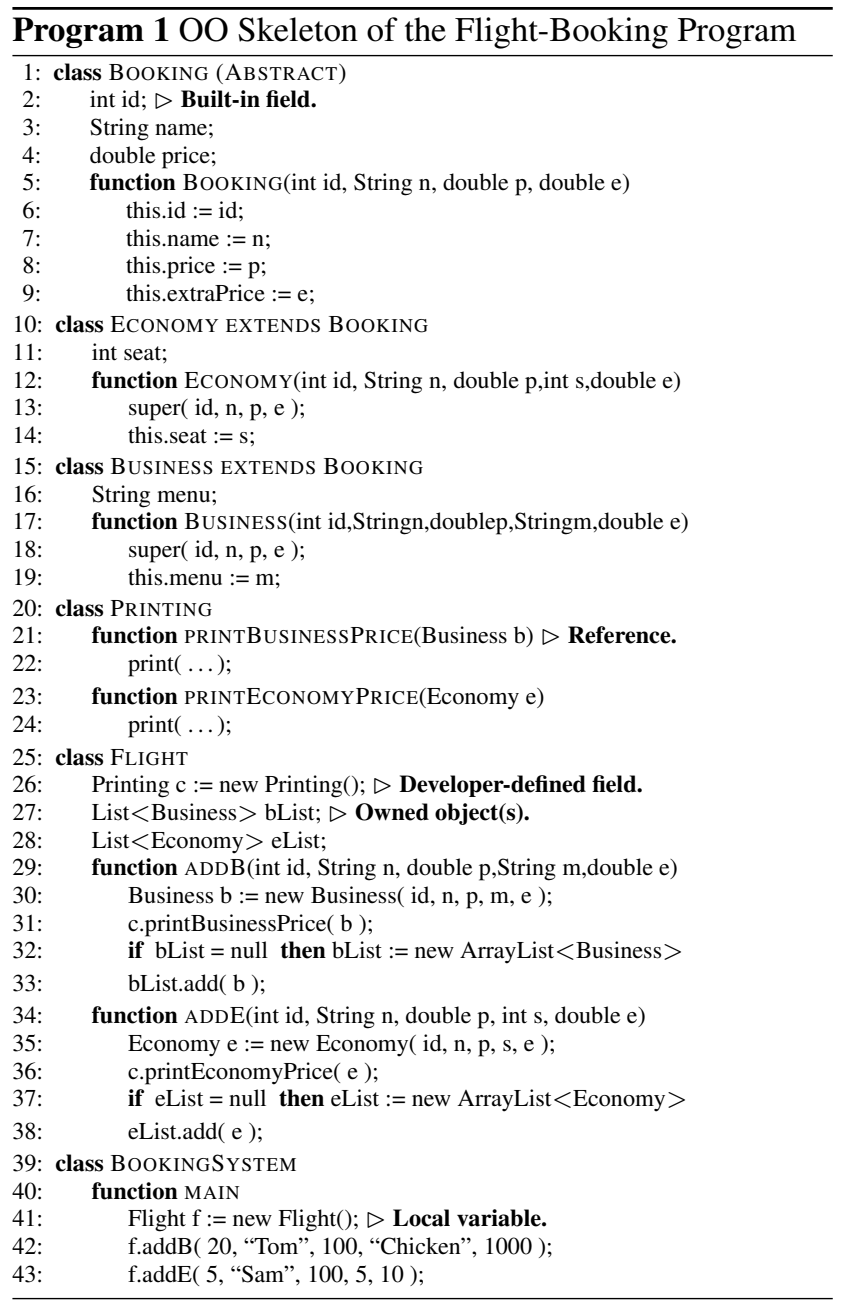

\section{Object-Oriented Relationships}

We illustrate our definitions via using a running example. We take an example that corresponds to a small part of an $\mathrm{OO}$ flight-booking system. The program calculates the total price of a booking and prints out the overall booking information. The Java-like pseudo-code of the classes of the above program is provided in Prog. 1.

Classifier fields and methods. A classifier mainly consists of classifier-level fields (e.g., built-in data-types, objects of other classifiers) and/or methods.

Owned object reference. An A classifier can be associated to an object of a B classifier even if $\mathrm{A}$ has not created the 
$B$ object. In this case, $A$ is associated with a reference to the B object (line 21 of Prog. 1). If A does not create a B object but $A$ has a reference to the object that is kept in the fields of A, then A has an owned reference to the object. To distinguish the case of an object reference owned by the classifier that created the object, we further use the term owned $o b$ ject. If $A$ creates a $B$ object stored in the fields of $A$, then $A$ has an owned object (line 27 in Prog. 1). The definitions of the concepts of owned object and reference are provided in the remainder of this section.

Object finalization. By default, all the references to an object are freed when a program finishes its execution. A classifier method may explicitly finalize an object via using a reference to the object before the termination of the program. If an A classifier has a reference to a B object and this reference has been finalised by another classifier, then $A$ has lost/cannot refer to the B object.

Definition 1 (OO Classifier) A classifier, $c$, includes ( $i$ ) its name $n$ whose prefix is its package path (this combination can uniquely identify the classifier in a program); (ii) its kind $k$ (concrete class, abstract class, interface, enum); (iii) $a$ (possibly empty) set of generic classifiers $g_{i}$ that $c$ extends/implements; (iv) a (possibly empty) set of classifierlevel developer-defined $f_{i}$ fields (owned objects), along with their maximum $l_{i}$ multiplicity; (v) a (possibly empty) set of the $d_{i}$ classifiers whose object references are explicitly finalized by c; (vi) a (possibly empty) set of the $r_{i}$ object references that are owned by c; (vii) a (possibly empty) set of the methods of c; (viii) a (possibly empty) set of the $u_{i}$ classifiers (along with their maximum $l_{i}$ multiplicity) whose objects are created by $c$. If the object is created by using a combination of generic and concrete classifiers, then the $u_{i}$ set includes both the generic and the concrete classifiers.

$c=\left(n, k,\left\{g_{i}\right\},\left\{\left(f_{i}, l_{i}\right)\right\},\left\{d_{i}\right\},\left\{r_{i}\right\},\left\{m_{i}\right\},\left\{\left(u_{i}, l_{i}\right)\right\}\right)$

Definition 2 (Method) A method is characterized by (i) its name $n$; (ii) a (possibly empty) set of arg $_{i}$ arguments that are developer-defined classifiers (along with their maximum $l_{i}$ multiplicity); (iii) its (possibly absent) ret developerdefined return type (along with its maximum l multiplicity).

$$
m=\left(n,\left\{\left(\arg _{i}, l_{i}\right)\right\},(\text { ret }, l)\right)
$$

Definition 3 (Owned Object) A $c_{1}$ classifier owns an object of a $c_{2}$ classifier if the $c_{2}$ object belongs to the developer-defined fields of $c_{1}$ and the $c_{2}$ object has been created by the $c_{1}$ classifier. To put it formally, a $c_{2}$ object is owned by $c_{1}$ if the following condition is evaluated as true.

$$
\operatorname{ownedObj}\left(c_{2}, c_{1}\right):=c_{2} \in c_{1} \cdot\left\{f_{i}\right\} \wedge c_{2} \in c_{1} \cdot\left\{u_{j}\right\}
$$

Definition 4 (Owned Reference) A $c_{1}$ classifier just owns a reference to an object of a $c_{2}$ classifier if the $c_{2}$ object reference belongs to the developer-defined fields of $c_{1}$ but the $c_{2}$ object has not been created by the $c_{1}$ classifier.

$$
\text { ownedRef }\left(c_{2}, c_{1}\right):=c_{2} \in c_{1} \cdot\left\{f_{i}\right\} \wedge c_{2} \notin c_{1} \cdot\left\{u_{j}\right\}
$$

Definition 5 (Associated Reference) $A c_{1}$ classifier is associated with a reference to an object of a $c_{2}$ classifier if the $c_{2}$ reference does not belong to the developer-defined fields of $c_{1}$, the $c_{2}$ object has not been created by the $c_{1}$ classifier, and the $c_{2}$ object is included in the arguments of a method of the $c_{1}$ classifier.

$$
\begin{gathered}
\operatorname{assocRe}\left(c_{2}, c_{1}\right):=c_{2} \notin c_{1} \cdot\left\{f_{i}\right\} \wedge \\
c_{2} \notin c_{1} \cdot\left\{u_{j}\right\} \wedge c_{2} \in c_{1} \cdot m_{k} \cdot\left\{\arg _{l}\right\}
\end{gathered}
$$

\section{OO and UML Relationship Mapping}

According to [15], composition should be defined based on the lifetime and the share-ability properties. The shareability property requires that an object of a classifier, along with the references to the object, must be owned by at most one composite classifier. The lifetime property requires that the object of a composite classifier cannot be outlived by its owned objects. In other words, when the object of a composite classifier is finalized, its owned objects and the references to the owned objects are finalized too.

Definition 6 (Object Share-ability) $A c_{1}$ classifier shares a $c_{2}$ object with a $c_{3}$ classifier if there is a reference owned by $c_{3}$ to the $c_{2}$ object that is created and owned by $c_{1}$.

$\operatorname{share}\left(c_{1}, c_{2}, c_{3}\right):=$ ownedObj $\left(c_{2}, c_{1}\right) \wedge$ ownedRef $\left(c_{2}, c_{3}\right)$

To compare the lifetime between an object of a composite classifier and its owned objects via using OO relationships, we define and prove the following theorem that is based on the object share-ability.

Theorem 1 (Composite object lifetime) The lifetime of an object of a $c_{1}$ composite classifier is longer than or the same to the lifetime of an object of a $c_{2}$ classifier that is owned by $c_{1}$ if there is no other $c_{3}$ classifier that explicitly finalizes the $c_{2}$ object and $c_{3}$ does not own a reference to the $c_{2}$ object. If $c_{3}$ owns a reference to the $c_{2}$ object, then $c_{1}$ should explicitly finalize $c_{2}$.

$$
\begin{array}{r}
\text { life }\left(c_{1}, c_{2}\right):=\text { ownedObj }\left(c_{2}, c_{1}\right) \wedge c_{2} \notin c_{3} \cdot\left\{d_{i}\right\} \\
\left(\nexists c_{3}: \text { ownedRef }\left(c_{2}, c_{3}\right) \vee c_{2} \in c_{1} \cdot\left\{d_{i}\right\}\right)
\end{array}
$$

Proof 1 We assume that a $c_{2}$ object is owned by a $c_{1}$ object and we examine all the possible cases with respect to the ownership of the $c_{2}$ object/references and the finalization time of the objects. 
(a) If $c_{3}$ explicitly finalizes $c_{2}$, then $c_{1}$ cannot use its owned $c_{2}$ and consequently, the lifetime comparison of $c_{1}$ and $c_{2}$ is meaningless (the second condition is false).

(b) If $c_{3}$ does not explicitly finalize $c_{2}, c_{3}$ owns a reference to $c_{2}$, and

(i) $c_{1}$ is finalized without finalizing $c_{2}$ (swallow finalization), then $c_{2}$ has longer lifetime than $c_{1}$ because there is a live reference to $c_{2}$ in the $c_{3}$ object (both third and fourth conditions are false)

(ii) $c_{1}$ and $c_{2}$ are finalized together (deep finalization), then $c_{1}$ and $c_{2}$ have the same lifetime and $c_{3}$ cannot use $c_{2}$ because $c_{2}$ has been finalized (the first, second, and fourth conditions are true).

(c) If $c_{3}$ does not explicitly finalize $c_{2}$, if there is no $c_{3} o b$ ject that owns reference(s) to $c_{2}$ and

(i) $c_{1}$ is finalized without finalized $c_{2}$ (swallow finalization), then there is no left object that uses $c_{2}$ and we consider that the lifetime of $c_{1}$ and $c_{2}$ is the same (the first, second, and third conditions are true)

(ii) $c_{1}$ and $c_{2}$ are finalized together (deep finalization), then $c_{1}$ and $c_{2}$ have the same lifetime (all conditions are true).

Illustrative example. The Flight object owns a Business object in Prog. 1, but there is no reference to the same Business object owned by another object. According to Theorem 1, the lifetime of the Flight object is longer or the same to the lifetime of the Business object.

Definition 7 (Composition) A c classifier is composed by a $c_{2}$ classifier if there is no $c_{3}$ classifier that shares with the $c_{1}$ classifier the same $c_{2}$ object and the lifetime of the $c_{1}$ object is longer or the same to the lifetime of the $c_{2}$ object.

$\operatorname{comp}\left(c_{1}, c_{2}\right):=\nexists c_{3}: \operatorname{share}\left(c_{1}, c_{2}, c_{3}\right) \wedge \operatorname{life}\left(c_{1}, c_{2}\right)$

Illustrative example. The Flight object in Prog. 1 owns a Business object, there is no reference to the same Business object that is owned by another object, and the Flight and the Business objects have the same lifetime. In this case, the Flight and the Business classes have a UML composition relationship.

Aggregation relates a composite classifier and its owned objects/references. To capture this relationship, we use the owned object and reference relationships (Def. 3 and Def. 4), without the composite and the owned objects/references satisfying the lifetime and the share-ability properties.
Definition 8 (Aggregation) $A c_{1}$ classifier aggregates a $c_{2}$ classifier if $c_{1}$ owns $c_{2}$ object $(s) /$ reference $(s)$ but $c_{1}$ does not have a composition relationship with $c_{2}$.

$$
\begin{gathered}
\operatorname{aggr}\left(c_{1}, c_{2}\right):=\left(\text { ownedObj }\left(c_{1}, c_{2}\right) \vee\right. \\
\text { ownedRef } \left.\left(c_{1}, c_{2}\right)\right) \wedge ! \operatorname{comp}\left(c_{1}, c_{2}\right)
\end{gathered}
$$

According to the UML standard, association exists when a classifier is associated with references to object(s) of another classifier. In other words, the association can be defined by using Def. 4. But if the former classifier is composite that owns the object(s)/reference(s) of the latter classifier, then the classifiers may have a composition/aggregation relationship.

Definition 9 (Association) $A c_{1}$ classifier is associated with a $c_{2}$ classifier if a $c_{1}$ object does not own a $c_{2} o b$ ject/reference and the $c_{1}$ object is associated with a reference to a $c_{2}$ object: $\operatorname{assoc}\left(c_{1}, c_{2}\right):=\operatorname{assocRe} f\left(c_{1}, c_{2}\right)$

Please note a set of binary associations can be combined to form $\mathrm{N}$-ary associations that may exist. However, the current work focuses on the recovery of binary associations, leaving as future work the recovery of $\mathrm{N}$-ary associations.

Definition 10 (Realization) $A \quad c_{1}$ classifier realizes a $c_{2}$ classifier if $c_{2}$ is an interface and $c_{1}$ implements $c_{2}$.

$$
\operatorname{impl}\left(c_{1}, c_{2}\right):=c_{2}=c_{1} \cdot g_{i} \wedge c_{2} \cdot k=\text { "interface" }
$$

Definition 11 (Inheritance) A $c_{1}$ classifier inherits from a $c_{2}$ classifier if $c_{1}$ extends $c_{2}$ and $c_{2}$ is concretelabstract class: inher $\left(c_{1}, c_{2}\right):=c_{2}=c_{1} . g_{i} \wedge c_{2} . k=$ "class"

The dependency generally indicates that a source classifier uses an object of a target classifier. But if the former is a composite classifier that owns the object(s)/reference(s) of the latter, then the classifiers have a composition/aggregation relationship. Otherwise, if the former uses a reference to an object of the latter, then the classifiers have an association relationship.

Definition 12 (Dependency) $A \quad c_{1}$ classifier depends on

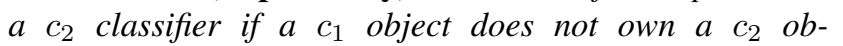
ject/reference, the $c_{1}$ object is not associated with a $c_{2} o b-$ ject reference, and $c_{2}$ is the return type of a $c_{1}$ method or $c_{1}$ has created the $c_{2}$ object.

$$
\begin{array}{r}
\operatorname{dep}\left(c_{1}, c_{2}\right):=\text { ! ownedObj }\left(c_{1}, c_{2}\right) \wedge \text { ! ownedRef }\left(c_{1}, c_{2}\right) \wedge \\
\quad \text { assocRef }\left(c_{1}, c_{2}\right) \wedge\left(c_{2} \in c_{1} \cdot\left\{u_{i}\right\} \vee c_{2}=c_{1} . m_{j} . \text { ret }\right)
\end{array}
$$

Overall example. Applying our definitions on Prog. 1, we took as output the UML class diagram of Fig. 2 . On the contrary, the diagram generated by the professional ObjectAid UML recovery tool is presented in Fig. 1. Comparing the two diagrams, we observe that the diagrams differ in five out of the seven UML relationship arrows. 
Table 1. The dataset that we used for the effectiveness evaluation of the UML Recoverer.

\begin{tabular}{|c|c|c|c|c|c|c|c|c|c|c|c|c|}
\hline ID & \multicolumn{5}{|c|}{ Num. of Classifiers } & \multicolumn{2}{|c|}{ Num. of Fields } & \multirow{2}{*}{$\begin{array}{l}\text { Num. of } \\
\text { Methods }\end{array}$} & \multicolumn{2}{|c|}{ Num. of Method Arguments } & \multicolumn{2}{|c|}{ Num. of Method Return-Types } \\
\hline & Total & Concrete & Abstract & Interface & Enum. & Total & Dev. defined & & Total & Dev. defined & Total & Dev. defined \\
\hline 1 & 15 & 15 & 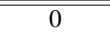 & 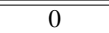 & 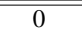 & 27 & 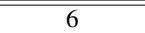 & 33 & 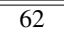 & 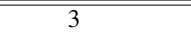 & 11 & "0 \\
\hline 2 & 23 & 23 & 0 & 0 & 0 & 79 & 40 & 112 & 64 & 36 & 68 & 19 \\
\hline 3 & 23 & 13 & 1 & 9 & 0 & 66 & 16 & 92 & 90 & 12 & 22 & 0 \\
\hline 4 & 30 & 29 & 1 & 0 & 0 & 69 & 38 & 102 & 60 & 17 & 48 & 6 \\
\hline 5 & 31 & 21 & 7 & 3 & 0 & 54 & 16 & 154 & 123 & 43 & 97 & 12 \\
\hline 6 & 36 & 30 & 2 & 4 & 0 & 48 & 20 & 168 & 124 & 36 & 128 & 20 \\
\hline 7 & 34 & 23 & 2 & 9 & 0 & 26 & 8 & 89 & 81 & 36 & 33 & 6 \\
\hline 8 & 44 & 35 & 3 & 6 & 0 & 99 & 58 & 216 & 107 & 40 & 112 & 25 \\
\hline
\end{tabular}

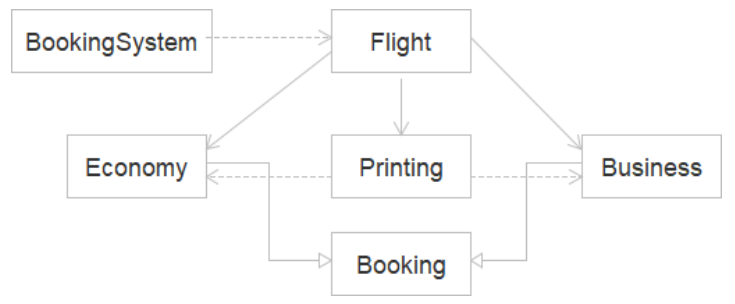

Figure 1. The diagram recovered by ObjectAid for Prog. 1.

\section{Experimental Evaluation}

We implemented in Java the UML Recoverer researchprototype of our approach. We evaluate the effectiveness of the UML Recoverer on anonymized student projects against ground-truth UML diagrams and diagrams recovered by existing professional UML class recovery tools. The number of the classifiers of the projects ranges from 15 to 44 (Table 1) and the number of their UML relationships ranges from 20 to 170 relationships. Searching for existing (free to use) UML class recovery Eclipse plug-ins in the Eclipse Marketplace, we found that the most widely used tools currently are the ObjectAid ${ }^{7}$ and the UML $\mathrm{Lab}^{8}$. To assess the effectiveness of the recovered binary UML relationships, we compare them against manually extracted relationships via using the precision and recall metrics [16].

The precision results are depicted in the first chart of Fig. 3. We observe the precision of the UML Recoverer steadily equals 1.0 in all projects (independently of the project cases). On the contrary, the precision of the other tools ranges from 0.37 to 0.86 and from 0.04 to 0.53 , respectively. The recall results are depicted in the second chart of Fig. 3. We observe the recall of the UML Recoverer ranges from 0.79 to 1.0. In particular, the lower the number of the abstract classes and the interfaces a project includes, the higher the recall of the UML

\footnotetext{
7 https://www.objectaid.com/class-diagram

8 https: //www.uml-lab.com/en/uml-lab/videos/ reverse-egnineering
}

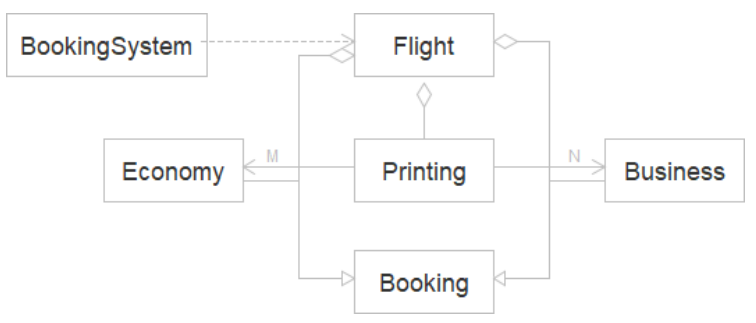

Figure 2. The diagram recovered based on our definitions for Prog. 1.

Recoverer is. This is due to the fact that the UML Recoverer does not capture association relationships to late-binding cases. The recall of the other tools ranges from 0.43 to 0.85 and from 0.05 to 0.53 , respectively.

To explain why the precision and the recall values of the two tools is very low in some cases, we inspected the numbers of the UML relationships recovered by the tools and we made the following observations. The two tools do not recover the aggregation and the composition relationships at all. In particular, the ObjectAid considers as dependencies/associations the relationships that are aggregations or compositions. The UML Lab considers as associations the relationships that are dependencies, aggregations or compositions. The number of the associations recovered by the UML Recoverer is slightly lower than the ground-truth number. The reason is the late binding to objects. In particular, there are methods in the student projects that accept as input objects of abstract classes/interfaces and the UML Recoverer identifies the association to abstract classes/interfaces but not to concrete classes.

\section{Conclusion and Future Work}

We formally defined a set of mappings between UML relationships and $\mathrm{OO}$ relationships via using static-code analysis exclusively. A future direction of our work is the comparison of our algorithm against UML class recovery approaches that apply dynamic-code analysis. Another inter- 

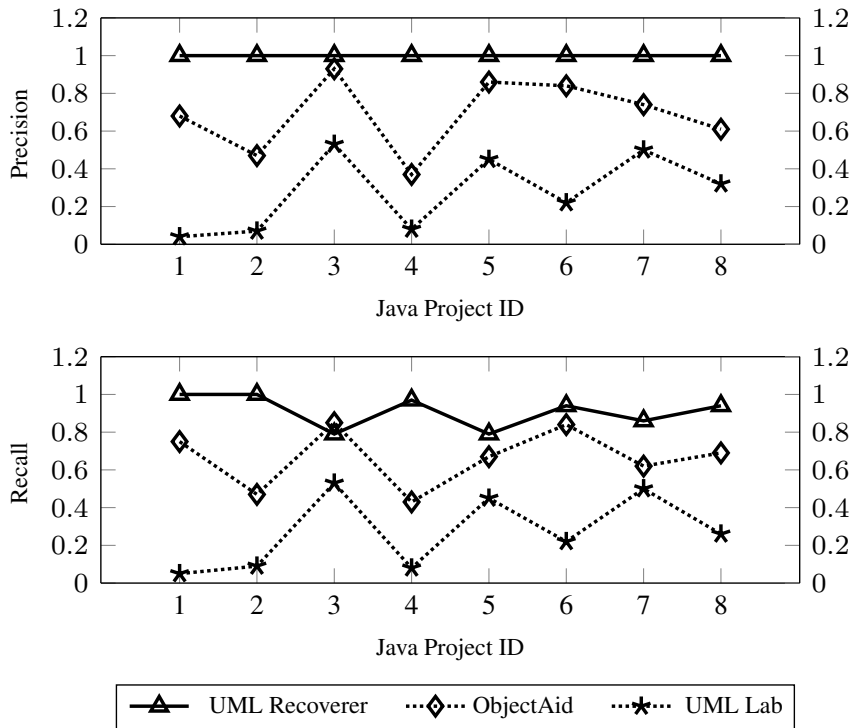

Figure 3. The precision and the recall results for the three recovery tools.

esting future direction would be the recovery of N-ary associations. Finally, the employment of semantic code analysis could further enrich the effectiveness of our approach.

\section{References}

[1] Y. Guéhéneuc and H. Albin-Amiot, "Recovering binary class relationships: Putting icing on the uml cake," in ACM SIGPLAN Conference on ObjectOriented Programming, Systems, Languages, and Applications, 2004, pp. 301-314.

[2] Y. Guéhéneuc, "A reverse engineering tool for precise class diagrams," in Conference of the Centre for Advanced Studies on Collaborative research. IBM, 2004, pp. 28-41.

[3] A. Milanova, "Composition inference for UML class diagrams," Automated Software Engineering, vol. 14, no. 2, pp. 179-213, 2007.

[4] W. Harrison, C. Barton, and M. Raghavachari, "Mapping UML designs to java," in ACM SIGPLAN Conference on Object-Oriented Programming Systems, Languages \& Applications, 2000, pp. 178-187.

[5] M. C. Leonardi, M. V. Mauco, L. Felice, G. Montejano, D. Riesco, and N. C. Debnath, "Recovering business process diagrams from UML diagrams," in IEEE International Conference on Computer Systems and Applications, 2010, pp. 1-6.
[6] H. M. Chavez, W. Shen, R. B. France, B. A. Mechling, and $\mathrm{G}$. $\mathrm{Li}$, "An approach to checking consistency between UML class model and its java implementation," IEEE Transactions on Software Engineering, vol. 42, no. 4, pp. 322-344, 2016.

[7] D. Torre, Y. Labiche, M. Genero, and M. Elaasar, "A systematic identification of consistency rules for UML diagrams," Journal of Systems and Software, vol. 144, pp. 121-142, 2018.

[8] D. Torre, Y. Labiche, M. Genero, M. T. Baldassarre, and M. Elaasar, "UML diagram synthesis techniques: a systematic mapping study," in ACM International Workshop on Modelling in Software Engineering, MiSE@ICSE, 2018, pp.33-40.

[9] D. Torre, Y. Labiche, M. Genero, M. Elaasar, and C. Menghi, "UML consistency rules: a case study with open-source UML models," in ACM International Conference on Formal Methods in Software Engineering, 2020, pp. 130-140.

[10] M. Grechanik, K. S. McKinley, and D. E. Perry, "Recovering and using use-case-diagram-to-source-code traceability links," in ACM SIGSOFT International Symposium on Foundations of Software Engineering, 2007, pp. 95-104.

[11] J. Niere, "Recovering uml diagrams from java code using patterns," in Workshop on Soft Computing Applied to Software Engineering, 2001, pp. 1-9.

[12] M. J. Decker, K. Swartz, M. L. Collard, and J. I. Maletic, "A tool for efficiently reverse engineering accurate UML class diagrams," in IEEE International Conference on Software Maintenance and Evolution, 2016, pp. 607-609.

[13] A. M. Sutton and J. I. Maletic, "Mappings for accurately reverse engineering UML class models from $\mathrm{C}++$," in IEEE Working Conference on Reverse Engineering, 2005, pp. 175-184.

[14] A. Milanova, "Precise identification of composition relationships for UML class diagrams," in IEEE/ACM International Conference on Automated Software Engineering, 2005, pp. 76-85.

[15] H. M. Chavez and W. Shen, "Formalization of UML composition in OCL," in IEEE International Conference on Computer and Information Science, 2012, pp. 675-680.

[16] R. A. Baeza-Yates and B. A. Ribeiro-Neto, Modern Information Retrieval. ACM Press/Addison-Wesley, 1999. 